\title{
Proteomics in Clinical Studies and its Applications
}

\section{Chiu Hung Chuan* \\ Department of Medical Genetics, Children Hospital Building, National Taiwan University Hospital, Taiwan, China}

The field of clinical proteomics has rapidly evolved during the past few years and is continuously growing as new methodologies and technologies emerge. The aim of this article is to introduce the field of proteome analysis as well as its applications to human disease biomarker discovery.

Human body contains approximately 1 million of proteins that are products of over 20 thousands of genes in the human genome. The expression of functional proteins involves many posttranslational modifications. One of them is the phosphorylation. The phosphorylation of proteins is important in the regulation of most cellular responses to external stimuli [1]. The analysis of phosphorylated proteins and the characterization of phosphorylation sites under different biological conditions are necessary to understand the complex signalling networks that regulate all major cellular processes. For example, Signal transducer and activator of transcription 3 (Stat3) is phosphorylated on Tyr705 and forms dimmers through phosphotyrosine-Src homology 2 domain interactions [2]. The dimerized Stat 3 molecules enter the nucleus and bind to a consensus DNA sequence in the promoters of its target genes to regulate transcription [3].

Since proteins given cellular structure and biological function, to understand the physiologic and pathophysiologic processes, the whole proteome should be studied. Proteome has been defined as "proteome complement of the genome" related to all proteins of the prokaryotes or the eukaryotes. Most proteins act in complexes and form a very complex interaction network. Some proteins interact only with the few others. The proteins of subcellular localization interact mostly with others of the same localization. Failures in protein interaction network, such as gain or loss of interaction, lead to human diseases. Proteomic approach enables the study of proteomes involving the global analysis of protein expression profiles, the identification of them and their function in any organ, tissue, cell or cell organelle. Modern proteomics, by its ability to detect dynamic changes in protein expression, localization and modification, has become a powerful tool to map signal transduction pathways and deliver the functional information that will promote insights in cell biology and systems biology.

Over the past decade a great progress in technologies to study cellular proteins has been made that may have potential applications in oncology and other human diseases. Proteomic technologies are used for protein profiling, identification and quantification in tissues, microdissected cells and body fluids. This approach allows to elaborate the putative biomarkers for a variety of human diseases. Experimental approaches involve the application of two-dimensional electrophoresis (2D-PAGE), multi-dimensional liquid chromatography, MALDI-TOF MS (Matrix-Assisted Laser Desorption Ionization Time-Of-Flight Mass Spectrometry), SELDI-TOF-MS (Surface-Enhanced Laser Desorption Ionization Mass Spectrometry) and protein/antibody arrays [4], as well as the bioinformatic and statistical tools pertinent to the analysis of proteomics data.

Using 2D-PAGE is limited because of very wide concentration range of proteins present in biological samples such as serum [5]. Proteins present in high concentration "mask" those potential biomarkers of diseases that are present only in trace amounts thus the new technologies are used that allow to capture selectively those proteins from the biological material that occur in the highest concentrations. MALDI-TOF MS is based on desorption and ionization of peptide fragments attached to the matrix surface by the short impulse of laser light and then analysis of ionic beam by mass spectrometry. SELDITOF-MS technology enables initial concentration of biological material, purification from interfering substances such as desalting, separation of different fractions and then separate analysis of peptide patterns [6].

Protein biochip array technology uses a solid matrix with specific ligands (antibodies, antigens) attached at pre-defined sites on the surface. At present, over 20 test regions can be pre-fabricated onto the biochip surface but the capacity of tests per biochip can increase several times. This technology utilizes the basic principles of immunology: competitive, sandwich or antibody capture immunoassays. The chemiluminescent signals are simultaneously measured for the full array of tests on each chip. In this technology a cellular proteome is immobilized on a matrix with subsequent immunodetection of total and activated forms of cell signaling proteins. The intensity of signals generated by the protein spots is correlated with biological and clinical information as diagnostic and prognostic indicators.

Proteomic approach allows monitoring of disease process by simultaneous analysis of hundreds of proteins or peptides in the human body fluids that leads to discovery of clinically relevant combinations of disease biomarkers. Analysis of serum protein profiles and protein interaction networks generates enormous amount of data that are stored in large data bases, at present mostly available online

Recently, Juan et al. [7] described some proteomics to the discovery and establishment of powerful diagnostic tools (e.g., Protein Chip array) in the management of cancer. The evolution in proteomic technologies has not only enabled the screening of a large number of samples but also enabled the identification of pathologically significant proteins, such as phosphoproteins, and the quantitation of difference in protein expression under different conditions. Multiplexed assays are used widely to accurately fractionate various complex samples such as blood, tissue, cells, and Helicobacter pylori-infected specimens to identify differentially expressed proteins. Biomarker detection studies have substantially contributed to the areas of secretome, metabolome, and phosphoproteome.

Recent data on proteomics have shown the wide clinical applications: in the study of inflammatory bowel diseases, in the diagnosis and prognosis of different types of cancer (breast, lung,

*Corresponding author: Chiu Hung Chuan, PhD, Department of Medical Genetics, Children Hospital Building, National Taiwan University Hospital, Taiwan, China, Tel: 886-2-23123456 ext 71904; E-mail: gaki98@yahoo.com.tw

Received May 22, 2012; Accepted May 22, 2012; Published May 25, 2012

Citation: Chuan CH (2012) Proteomics in Clinical Studies and its Applications. Single Cell Biol 1:e108. doi:10.4172/2168-9431.1000e108

Copyright: ( 2012 Colin VL. This is an open-access article distributed under the terms of the Creative Commons Attribution License, which permits unrestricted use, distribution, and reproduction in any medium, provided the original author and source are credited. 
ovarian, thyroid, liver), in the diagnosis and prognosis of sepsis and ischemic and traumatic brain injury, etc. The proteomic approach offers a powerful tool for discovery of novel more effective drugs that may be used for individualized therapy, especially in the treatment of cancer.

In spite of very high costs, the impact of modern advanced proteomic technologies for clinical diagnosis and prognosis of human diseases in the future remains undisputed.

\section{References}

1. Nita-Lazar A, Saito-Benz H, White FM (2008) Quantitative phosphoproteomics by mass spectrometry: past, present and future. Proteomics $8: 4433-4443$.
2. Becker S, Groner B, Muller CW (1998) Three-dimensional structure of the Stat3beta homodimer bound to DNA. Nature 394: 145-151

3. Seidei HM, Milocco LH, Lamb P, Darnell JE Jr, Rosen J, et al. (1995) Spacing of palindromic half sites as a determinant of selective STAT (signal transducers and activators of transcription) DNA binding and transcriptional activity. Proc Natl Acad Sci U S A 92: 3041-3045

4. Alex P, Gucek M, Li X (2009) Applications of proteomics in the study of inflammatory bowel diseases: Current status and future directions with available technologies. Inflamm Bowel Dis 15: 616-629

5. Dumnicka P (2007) Proteomics Badanie i diagnoza 13: 9-11

6. Drozdz R, Tisonczyk J (2007) From Tiselius to proteomics-New perspectives of urine protein analysis. In Vitro Explorer 6: 15-21

7. Lin LL, Huang HC, Juan HF (2012) J Proteomics. 